\title{
RNA interference and the use of small interfering RNA to study gene function in mammalian systems
}

\author{
I Bantounas, L A Phylactou' ${ }^{1}$ and J B Uney \\ The Henry Wellcome Laboratories for Integrative Neuroscience and Endocrinology, Dorothy Hodgkin Building, University of Bristol, Whitson Street, \\ Bristol BS1 3NY, UK \\ ${ }^{1}$ The Cyprus Institute of Neurology and Genetics, 6 International Airport Avenue, P.O. Box 3462, 1683 Nicosia, Cyprus
}

(Requests for offprints should be addressed to J B Uney; Email: james.uney@bristol.ac.uk)

\begin{abstract}
In the past 2 years, extraordinary developments in RNA interference (RNAi)-based methodologies have seen small interfering RNAs (siRNA) become the method of choice for researchers wishing to target specific genes for silencing. In this review, an historic overview of the biochemistry of the RNAi pathway is described together with the latest advances in the RNAi field. Particular emphasis is given to strategies by which siRNAs are used to study mammalian gene function. In this regard, the use of plasmid-based and viral vector-based systems to mediate long-term RNAi in vitro and in vivo are described. However, recent work has shown that non-specific silencing effects and activation of the interferon response may occur following the use of some siRNA and delivery vector combinations. Future goals must therefore be to understand the mechanisms by which siRNA delivery leads to unwanted gene silencing effects in cells and, in this way, RNAi technology can reach its tremendous potential as a scientific tool and ultimately be used for therapeutic purposes.
\end{abstract}

Journal of Molecular Endocrinology (2004) 33, 545-557

\section{Introduction}

A number of technologies have been used in an attempt to mediate the down-regulation of gene expression. For example, anti-sense oligonucleotides and ribozymes have been used for more than a decade to target specific RNAs for degradation. Although these methods worked satisfactorily in some simple experimental models, they have generally not delivered effective gene silencing in complex mammalian systems. However, in the past 2 years, extraordinary developments in RNA interference (RNAi)-based methodologies have seen small interfering RNAs (siRNAs) become the primary means by which most researchers attempt to target specific genes for silencing. RNAi was first discovered in Caenorhabditis elegans, when it was noted that introducing a double-stranded RNA (dsRNA) that was homologous to a specific gene resulted in the post-transcriptional silencing of that gene. The obvious therapeutic potential of RNAi resulted in rapid elucidation of its mechanism of action and it is now known that gene silencing is mediated via two main steps: (i) the dsRNA is initially recognised by an enzyme of the RNase III family of nucleases, named Dicer, and processed into small double-stranded molecules, termed siRNA and (ii) the siRNAs are bound by the RNA-induced silencing complex (RISG), which is a multi-protein complex (with RNase activity) that guides the targeted RNA to degradation. Although early investigations found that the introduction of long dsRNA had the potential to mediate the down-regulation of any gene, it was also found that in mammalian cells an anti-viral interferon response (IR) that resulted in the cessation of all protein synthesis was also elicited. Synthetic siRNAs (21-23 nucleotides in length) were shown not to elicit this IR and have hence been used in studies of mammalian gene function. More 
recently, plasmid and viral-based expression of small hairpin RNAs (shRNA) have been developed; these methods allow for longer-lived gene silencing effects and the use of viral vectors facilitates the use of RNAi in neuronal cells. Finally, comparing the activity of transfected siRNAs and virally delivered shRNAs shows that the efficacy of the RNAi effect is highly dependent on the cellular environment.

\section{The cell biology of the RNAi pathway}

The first step in the RNAi pathway involves the processing of large dsRNAs into small, 21-23 nucleotide long siRNA molecules (Zamore et al. 2000, Elbashir et al. 2001). Initial studies in Drosophila showed that an RNase III enzyme (known to recognise dsRNA and reviewed in Conrad \& Rauhut 2002) was responsible for this processing and that the siRNAs possessed $3^{\prime}$ hydroxyl and 5' phosphate groups and, importantly, a $3^{\prime}$ overhang of two unpaired nucleotides on each strand (Elbashir et al. 2001). A specific RNAase III enzyme was then found to be responsible for cleaving the dsRNAs and was named Dicer (Bernstein et al. 2001). The sequence homology and functional studies that followed led to the identification of Dicer homologues in Arabidopsis (Golden et al. 2002), Dictyostelium (Martens et al. 2002), fission yeast (Provost et al. 2002b), C. elegans (Ketting et al. 2001), mouse (Nicholson \& Nicholson 2002) and human (Provost et al. 2002a, Zhang et al. 2002).

A proposed model for the action of Dicer involves the ATP-dependent translocation of the enzyme along its dsRNA target. The efficiency with which Dicer cleaves a particular dsRNA molecule has also been shown to be directly proportional to the length of the target, since the longer the dsRNA, the greater the amount of siRNA produced and hence the more potent the silencing effect (Bernstein et al. 2001). This size limitation may prevent Dicer binding to small intramolecular base-paired regions of endogenous mRNAs. Human Dicer-mediated cleavage of dsRNA is thought to occur sequentially, beginning at the termini of the dsRNA, and by the excision of small dsRNA fragments of a defined length (Ketting et al. 2001, Zhang et al. 2002).

Following the cleavage of dsRNA into siRNAs by Dicer the second important stage of mRNA degradation occurs. This is mediated by a protein complex with nuclease activity known as RISC which is guided to its target RNA by siRNA (Hammond et al. 2000). This guide role of siRNA was proposed after the observation that dsRNA would only lead to the degradation of an mRNA with a homologous sequence, leaving the rest of the RNA in the cell unaffected. Moreover, it was shown that both siRNA and protein were required to mediate cleavage of the target (Hammond et al. 2000). Following the initial discovery of the existence of a ribonucleoprotein complex as a mediator of RNAi, the components and mechanism of action of RISG began to be elucidated and both inactive and active forms of RISC complex (the active termed RISC*) were found. Nykanen et al. (2001) found that a second ATP-dependent step was involved in the pathway and showed that following unwinding of the siRNA duplex, RISG was converted to RISC*. In a separate seminal study, RISC* was found to be associated only with the antisense strand of the siRNA (Martinez et al. 2002). Hence, although the siRNA needs to be double stranded in order to be efficiently recognised and bound to RISC, the two siRNA strands must unwind before RISC becomes active. Accordingly, it was concluded that either the RISG complex has ATP-dependent helicase activity or a helicase enzyme is associated with RISC. The efficient cleavage of the target mRNA by RISC was also shown to be dependent on the phosphorylation of the 5' siRNA duplex (Nykanen et al. 2001, Martinez et al. 2002).

In non-mammalian cells, there is evidence that an alternative branch of the RNAi pathway that results in the amplification of the original message can account for the efficiency of gene silencing (Sijen et al. 2001) (Fig. 1). In this case, the unwound siRNA no longer acts as a guide to bring RISC to the target mRNA but merely as a primer for an RNA-dependent RNA polymerase (RdRP), which uses the target mRNA as a template to produce new dsRNA. This can subsequently be recognised and cleaved by Dicer, thus re-entering the RNAi pathway and initiating a new round of silencing. Therefore, not only is the mRNA targeted via the specific oligonuclotide sequence (and hence gene expression silenced) but also new dsRNAs arising from the entire mRNA sequence are created and thus amplify the original RNAi trigger. Several RdRPs participating in RNAi have been identified 


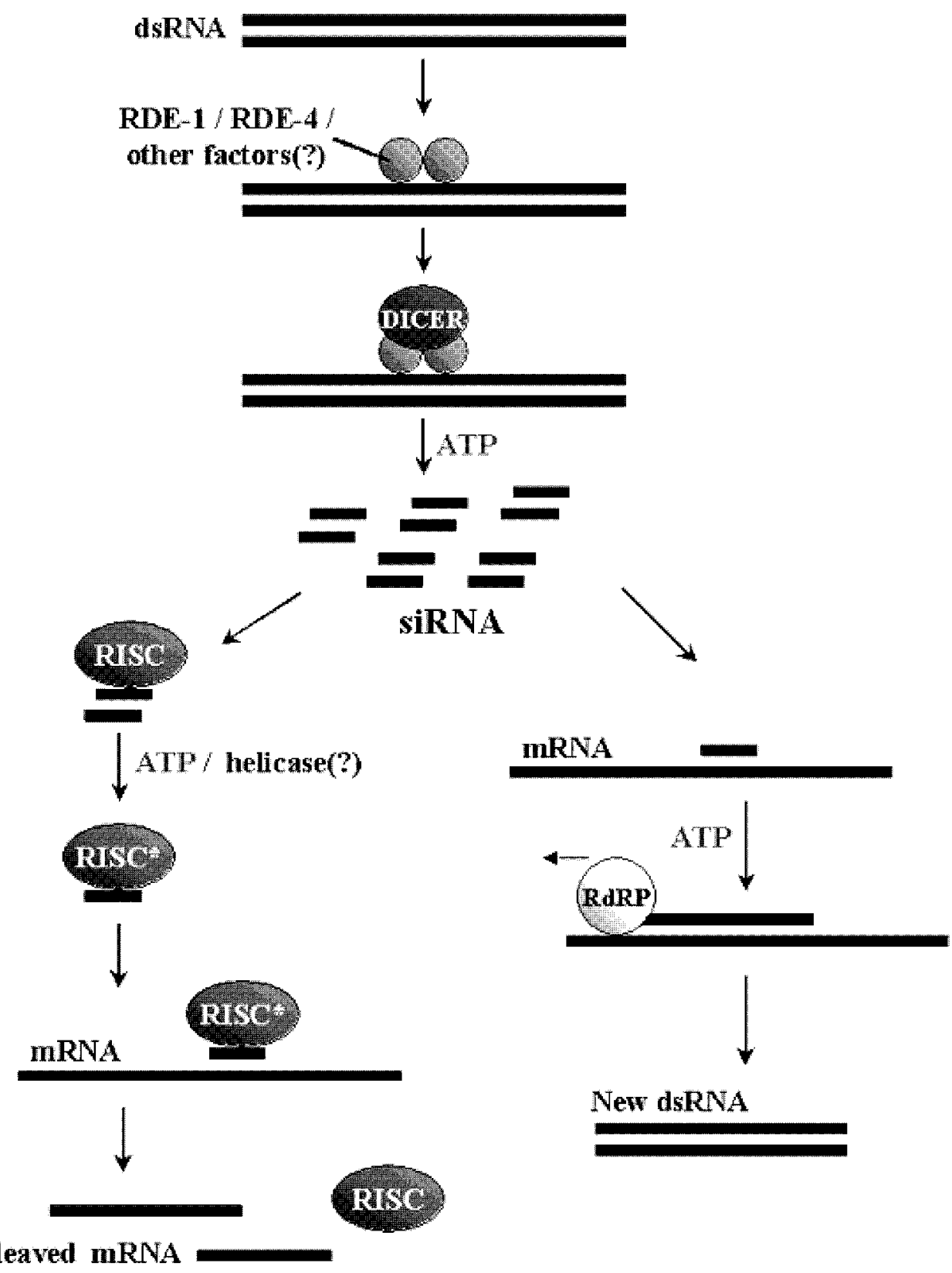

Figure 1 Overview of the RNAi pathway. When dsRNA enters the cell, it is recognised by the enzyme complex Dicer (with the aid of accessory protein factors, such as RDE-1 and $-4)$ and cleaved to yield siRNA. The siRNAs then bind to a multiprotein complex to form the RISC. Activation of the RISC is accompanied by the unwinding of the siRNA duplex, and one strand of the siRNA then guides RISC to the target mRNA (which is cleaved by RISC nuclease activity). Alternatively, in the cells of some organisms (such as C. elegans) the siRNA might also unwind, bind to the target mRNA and serve there as primers for an RdRP. RdRP extends the primer, so that the mRNA becomes double stranded and hence cleavable by Dicer, so as to initiate a new cycle of siRNA production. 
in fungi, plants and invertebrates (Cogoni \& Macino 1999, Dalmay et al. 2000, Mourrain et al. 2000, Smardon et al. 2000, Sijen et al. 2001, Martens et al. 2002). However, evidence to suggest that a similar amplification mechanism is present in mammalian cells has not yet been found.

\section{Gene inhibition due to microRNAs (miRNA)}

miRNAs are short RNA molecules that prevent gene expression by inhibiting translation (LagosQuintana et al. 2001, reviewed in Grosshans \& Slack 2002). The first two miRNAs to be studied were let-7 and lin-4, which control the expression of genes involved in developmental timing in $C$. elegans and Drosophila and hence were therefore also named small temporal RNAs (Lee et al. 1993, Olsen \& Ambros 1999, Reinhart et al. 2000). miRNAs are initially transcribed as hairpin structures (premiRNA) and are then processed to yield mature (single-stranded) miRNA (Grishok et al. 2001, Lagos-Quintana et al. 2001, Lau et al. 2001, Mourelatos et al. 2002). This maturation step was shown to be catalysed by Dicer in a number of organisms (Grishok et al. 2001, Hutvagner et al. 2001, Ketting et al. 2001), although with the aid of co-factors distinct from the RNAi pathway (Grishok et al. 2001, Tabara et al. 2002). The mature miRNA can then bind to the $3^{\prime}$-UTRs of their corresponding mRNAs, although their complementarity to the targets is not perfect as is the case for siRNA (Provost et al. 2002b). A RISG-like complex was also shown to participate in the miRNA pathway (Hutvagner \& Zamore 2002). The RNAi machinery - and especially RISG - is also thought to be involved in mediating hetrochromatic and transposon silencing (Ketting et al. 1999, Volpe et al. 2002, Schramke \& Allshire 2003). Dicer and RISC can therefore be perceived as two central points, where several molecular pathways controlling gene expression converge.

\section{Developing RNAi for use in mammalian cells}

\section{SiRNAs}

RNAi induced by long dsRNA has been used to study gene function in plants, worms and Drosophila. However, when dsRNAs more than 30 bp in length were used to study mammalian gene function it was noted that there was an inhibition of protein translation within the cell due to the activation of the interferon (IFN) system (one of the body's defences against viral infection) (Provost et al. 2002a). Over 100 cellular genes can be activated by IFNs and some encode enzymes that are dsRNA-binding proteins. This includes protein kinase $\mathrm{R}$ (PKR) which phosphorylates and inactivates eukaryotic initiation factor $2 \alpha$ and hence inhibits mRNA translation (Manche et al. 1992). Therefore, to apply RNAi technology to studies using mammalian systems (without inducing an IFN response) the silencing pathway had to be induced without the use of long dsRNA. Since synthetic 21-22 bp-long siRNAs could bypass the initial Dicer step, while retaining the ability to mediate gene silencing in Drosophila, they were used in studies using mammalian cells. Numerous studies have now shown that synthetic siRNAs can be used to silence both exogenous and endogenous gene expression in mammalian cells (Fig. 2). Synthetic siRNAs are now used in most investigations of gene expression and a number of methods of production are used (see below). At present, however, identifying the best target sequence is currently a process of trial and error, and a number of siRNAs must be tested against different sites on a particular mRNA. Nonetheless, it is recommended that the first 75-100 nucleotides of any mRNA be avoided as potential target sites, since they may contain protein-binding regulatory sequences $\left(5^{\prime}\right.$-UTR) that could interfere with the action of siRNA (Elbashir et al. 2002). Further studies to date have attempted to provide clues so as to rationalise siRNA design. Intriguingly, it was reported that sequence preference meant that only one strand of the siRNA duplex was incorporated into RISC* (Schwarz et al. 2003). It would therefore be desirable to design the siRNA duplex so that the antisense strand is the one to be preferentially used by RISC. Thus, it has been shown that when siRNA are thermodynamically unstable (A/U rich) or even contain mismatches towards the $5^{\prime}$ end of the antisense strand, then that strand is preferentially used by RISG resulting in more efficient silencing (Khvorova et al. 2003, Reynolds et al. 2004, Ui-Tei et al. 2004).

Although chemical synthesis provides a quick and easy way of obtaining siRNA, a more cost-effective 'do-it-yourself' method has been developed. It was based on the in vitro transcription 


\section{1-base target site}

\section{Target mRNA sequence}

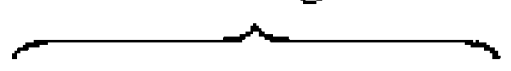

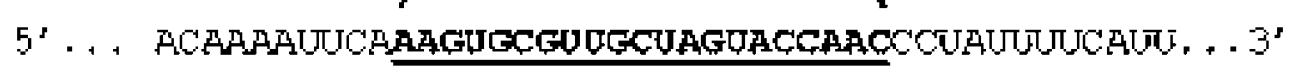

Double nudeotlde overkang

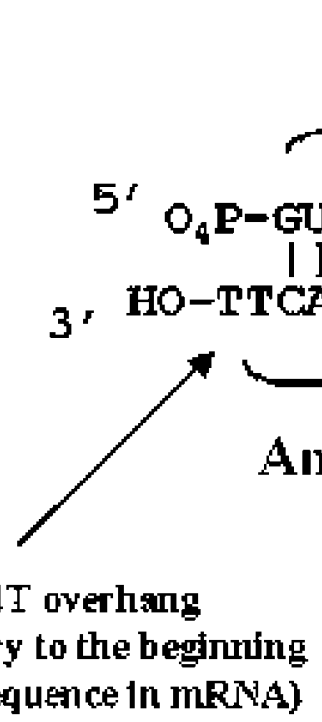

\section{Sense str and (21 bases)}

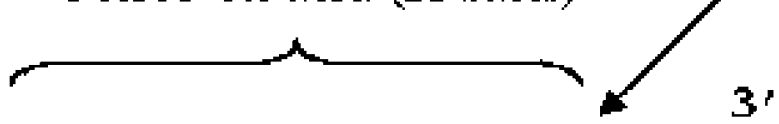

GUGCGUUGCUAGUACCAACTT-OH | ] | | | | | | | | | | | | | | | |

\section{Double dI overhang}

\section{(complementary to the beginuing of the target sequence in mRNA)}

Figure 2 Design of a synthetic siRNA. A target region is first chosen on the mRNA of interest (shown underlined). This region is optimally 21 nucleotides long (although siRNAs of 19-22 bp have also been successfully used) and it should begin with a double adenine residue (so as to be complementary to the double $U$ overhang of the siRNA antisense strand). The siRNA itself is double stranded (sense and antisense) comprising a 19 residue base-paired region with a double uridine, single-stranded overhang on each side of the siRNA. The antisense strand (which is actually used by RISC) is complementary to the target sequence. Note that the overhang of the sense strand does not correspond to the target sequence but is designed to be identical to the antisense strand overhang. In synthetic siRNA, the double $U$ overhangs are sometimes replaced by double $\mathrm{dT}$ to make the siRNA more stable in the cell. Once the siRNA enters the cell, the $5^{\prime}$ ends of each strand are phosphorylated and this is thought to be essential for siRNA-mediated silencing.

of the two siRNA strands, from DNA oligonucleotide templates (Donze \& Picard 2002) (see Fig. 3). These templates simply consisted of a sequence complementary to the T7 RNA polymerase promoter followed by a sequence complementary to either the sense or antisense siRNA strand. A second oligonucleotide consisting of the T7 promoter coding sequence was then annealed so that the T7 region became double stranded. This enabled the transcription of the sense or antisense siRNA strand by T7 RNA polymerase and, finally, the two transcribed strands were annealed to form the siRNA. siRNAs produced by this method have since been successfully used against a variety of
mRNA targets (Capodici et al. 2002, Sohail et al. 2003).

siRNAs are designed to be perfectly complementary to their targets and it was suggested that a one nucleotide difference between the antisense strand of the siRNA and the target mRNA could abolish siRNA activity (Chiu \& Rana 2002). However, it has also been demonstrated that, depending on the position of the mismatch, siRNA activity is affected to different degrees, and in some cases there is enough activity left to mediate significant silencing (Miller et al. 2003, Jackson et al. 2003). The degree of homology required between the siRNA and its target has been investigated using microarray 

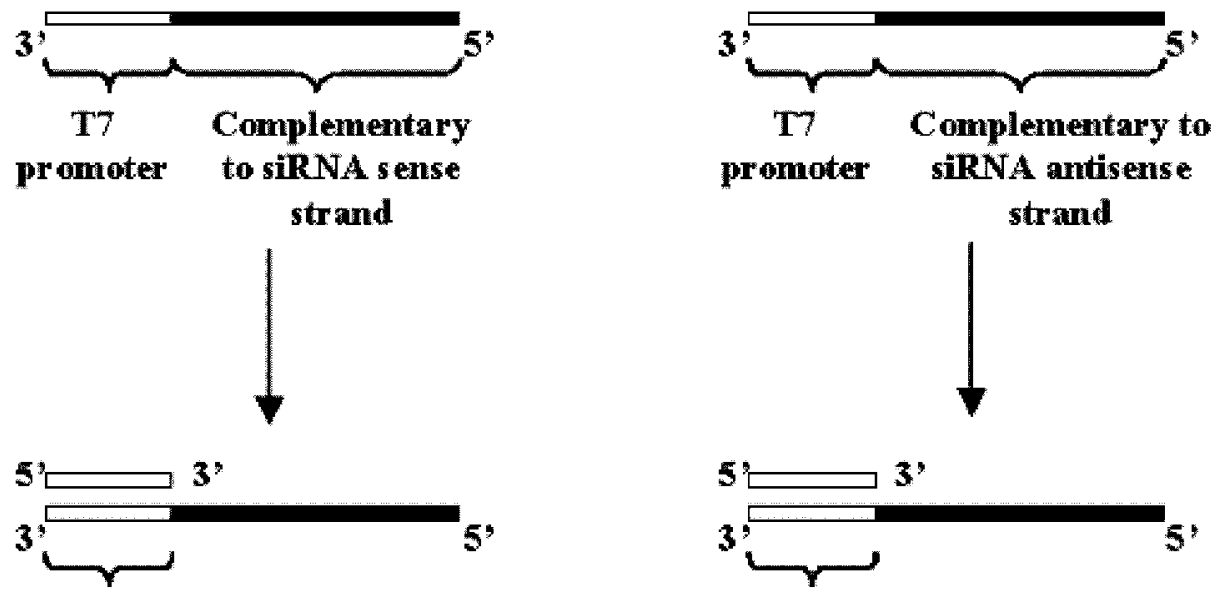

\section{Double-stranded}

T7 promoter
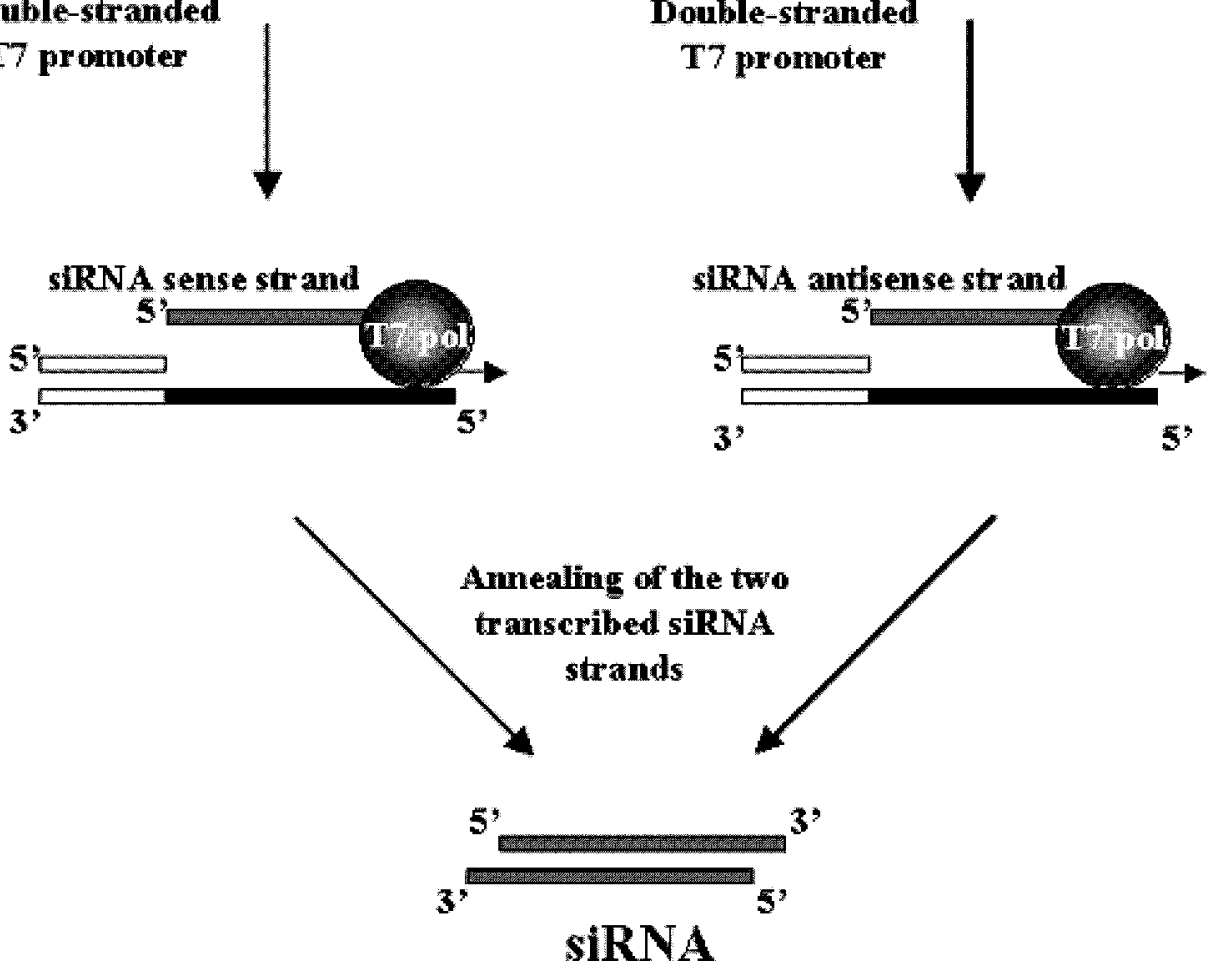

Figure 3 Construction of siRNA by T7 RNA polymerase-mediated in vitro transcription. Using this approach, transcription templates are first designed as deoxyoligonucleotides, each including a sequence complementary to the T7 promoter region, followed by a sequence complementary to either the sense or antisense siRNA strand. A separate oligonucleotide containing the T7 promoter sequence is then annealed to each template oligonucleotide. Transcription of each strand is subsequently driven in vitro by the now double-stranded T7 polymerase promoter, with the help of T7 polymerase. The two transcripts are finally annealed to each other to form the siRNA.

technology. In these studies, an siRNA designed against a specific gene was used to transfect cell lines and, subsequently, the expression of tens of thousands of genes was observed and compared with that of non-transfected controls. Jackson et al. (2003) reported that a number of genes were non-specifically down-regulated. Importantly, almost every one of these genes possessed regions 
that exhibited considerable (yet not perfect) homology to the siRNA and as little as 11 consecutive complementary nucleotides were in some cases enough to cause a non-specific effect. These findings could, at least in part, be explained by siRNAs being able to enter the miRNA pathway where perfect homology to the target mRNA is not necessary (Doench et al. 2003). Importantly, under certain conditions short single-stranded RNAs (ssRNAs) and siRNAs have now also been shown to activate the IFN system (Sledz et al. 2003, Kim et al. 2004). Furthermore, siRNAs expressed from both plasmid DNA and lentiviral vectors as hairpins (see below) were also shown to trigger an IFN response (Bridge et al. 2003). Intriguingly, Kim et al. (2004) found that removal of the $5^{\prime}$ triphosphate of the (ssRNA) transcripts prevented this activation and these findings therefore suggested that the IFN could be circumvented. The phosphorylation status of the $5^{\prime}$ and $3^{\prime}$ ends of the two strands of siRNA, although an important factor, has not been of major concern during the siRNA design. This is because commercially available siRNA have free hydroxyl groups at their $5^{\prime}$ ends, which are phosphorylated intracellularly. Moreover, synthetic siRNAs are designed to have a double uridine (or deoxy-thymidine) overhang at the $3^{\prime}$ end of each strand as this composition is the most efficient at mediating silencing (Elbashir et al. 2001). In summary, the optimal siRNA has a $19 \mathrm{bp}$ double-stranded region with 2- deoxy-thymidine/ uridine overhangs at each of its strands' 3 ' ends. Hence the target sequence always begins with two adenine residues (i.e. complementary to the double uridine overhang of the antisense strand of the siRNA).

\section{shRNAs}

Synthetic siRNAs do not have long half-lives once transfected into cells and inhibition of the target genes is correspondingly short lived. To increase the length of siRNA expression, eukaryotic polymerase III (PolIII) promoters were used to express siRNA from expression cassettes. The strategy (Fig. 4A) involved the cloning of a sequence coding for the sense strand of the siRNA of interest, followed by a spacer and then the equivalent to the anti-sense strand, which ended in a series of $5 \mathrm{U}$ residues. The inclusion of the spacer in the sequence mediated the formation of a hairpin structure, which allowed the sense and antisense sequences to form base pairs. The eukaryotic $\mathrm{H} 1$ and U6 PolIII promoters were used to drive expression of these shRNAs as they possess characteristics which make them suited to drive the expression of siRNAs: (i) they initiate from position +1 of the transcripts and (ii) the transcripts do not terminate with a poly-A tail but with a series of four to five thymidine residues, which results in a series of 3' U residues (Brummelkamp et al. 2002a). This means that a fully functional shRNA sequence can be transcribed since it will not contain any inhibitory $5^{\prime}$ nucleotide sequences and it can be designed to end in a uridine dimer. Indeed, the structure transcribed closely resembles the synthetic double-stranded siRNA, but with the two strands linked by a spacer sequence. This system has now been used to successfully inhibit gene expression in mammalian cells lines, with efficiencies comparable with that of synthetic siRNA. In a slightly different approach, the two siRNA strands were transcribed separately from two U6-based transcription cassettes included in the same or different vectors. The two strands were then shown to anneal within the cell to form an siRNA (see Fig. 4B) (Lee et al. 2002). Interestingly, it was reported that this method of expressing siRNA results in less potent gene silencing than when a hairpin siRNA (shRNA) is expressed (Miyagishi \& Taira 2003).

Experiments using PolIII promoter-based expression led to some interesting findings, suggesting optimal cassette design and mechanism of action. Thus, Brummelkamp et al. (2002a) showed that a nine base-long loop sequence mediated optimal transcript expression and that following expression the loop sequences were processed so that the siRNA strands were no longer connected by the loop region. This suggested that the active biological product was an siRNA. Other loop sequences and lengths have also been successfully used. For example, when an siRNA hairpin was designed so as to contain the miR-30 human miRNA loop (instead of a loop of random sequence) silencing activity significantly improved (Zeng et al. 2003, Boden et al. 2004). Furthermore, hybrid promoters have recently been developed aimed at improving or allowing regulated siRNA expression; in one study, the cytomegalovirus (CMV) enhancer sequence either upstream or downstream of the U6 promoter (Xia et al. 2003). 
(A)
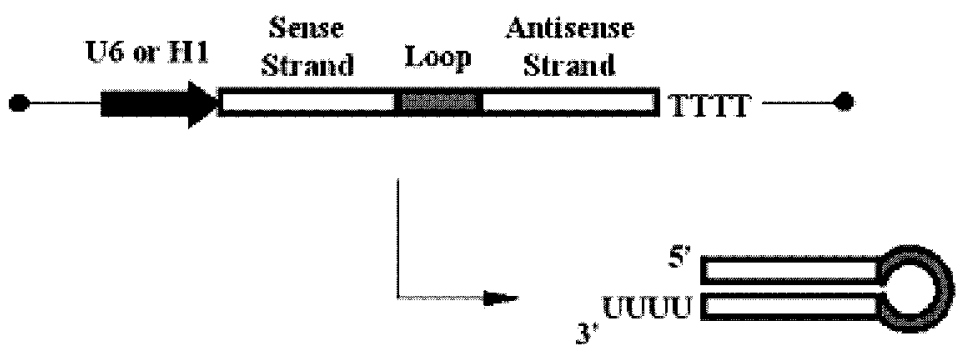

(B)

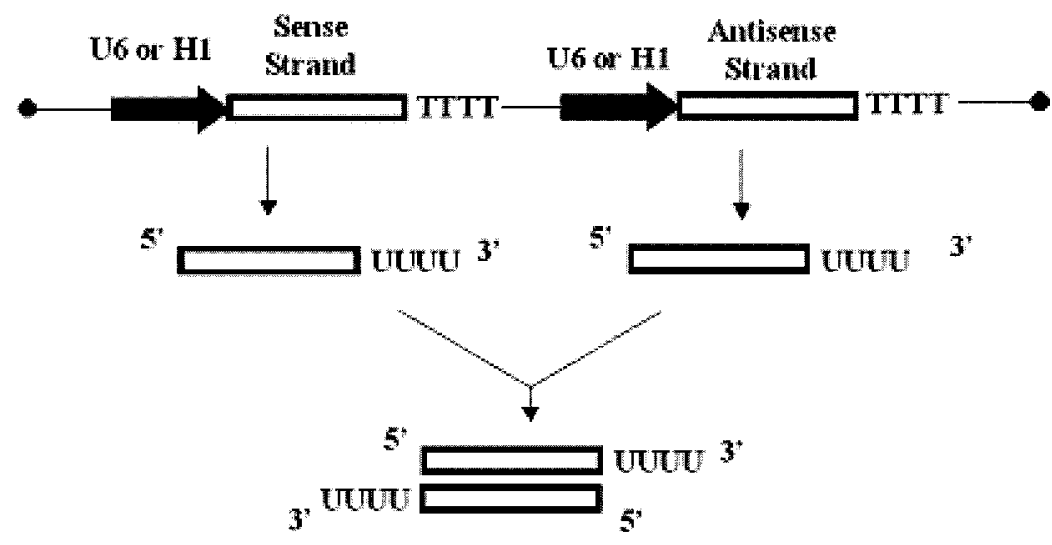

(C)

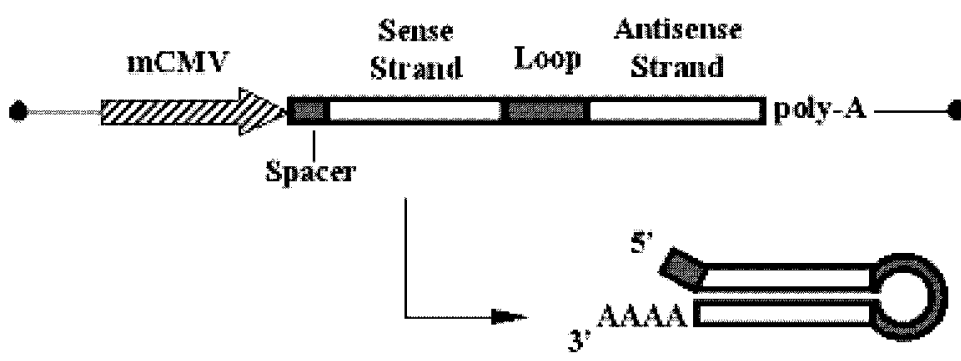

Figure 4 Basic siRNA expression cassettes. (A) U6 or H1 Pollll promoters drive the expression of a hairpin structure in which the sense and antisense strands of the siRNA are connected by a loop sequence. The addition of the oligo-T, Pollll transcription termination signal at the $3^{\prime}$ end of the cassette also ensures that the transcript has an oligo- $U$ overhang at its $3^{\prime}$ end. There is evidence that the transcribed hairpin is processed within the cell to give a genuinely double-stranded siRNA. (B) Two U6- or H1-based expression cassettes are used to drive the separate transcription of the sense and antisense strands in the cell. The two strands would then anneal to form a double-stranded siRNA. An oligo-U overhang is present on each strand. (C) A Polll promoter (a shortened version of the human CMV) drives the expression of a hairpin siRNA. The transcript includes extra nucleotides at its $5^{\prime}$ end and a poly-A tail (instead of an oligo- $U$ ) overhang at the $3^{\prime}$ end. 
In both cases - and regardless of the enhancer's orientation - the silencing of the target gene observed was significantly greater than that mediated by the unmodified U6 promoter. Furthermore, an inducible system for siRNA expression under the control of PolIII promoters has been developed (van de Wetering et al. 2003). In this case, the Tet operator (tet-O) sequence was used to replace a non-essential part of the $\mathrm{H} 1$ promoter. The resultant cassette was used to stably transfect cell lines. It was then shown that the expression of siRNA and hence silencing was dependent on the presence of tetracycline (or doxycycline) in the cells' culturing medium.

shRNA libraries that can also target every gene for silencing have been constructed recently. This technology involves ligating a large number of diverse shRNA-expressing inserts corresponding to a large number of genes to suitable vectors and then screening the resultant constructs for silencing activity (Berns et al. 2004, Hsieh et al. 2004, Miyagishi et al. 2004). Some research groups have also described the creation of siRNA libraries against specific genes (or genomes) where the shRNA-expressing inserts are derived from enzymatically processing the cDNA of the gene of interest (or a cDNA library respectively). The result is the creation of multiple vectors expressing shRNAs against target sites spanning the length of the gene (or genome) so that the most efficient ones can subsequently be selected (Luo et al. 2004, Sen et al. 2004, Shirane et al. 2004).

\section{Virally-mediated delivery of shRNAs}

Viral vectors carrying siRNA expression cassettes have been developed in an attempt to achieve delivery to a range of cell types (including neurones) and longer-term expression, leading to a more persistent silencing effect. Adenoviral vectors expressing siRNAs from a PollI-based promoter (Xia et al. 2002) and PolIII promoters have been used leading to the reduction of p53 levels in MCF-7 and A549 cells (Shen et al. 2003). Recently, an adeno-associated viral vector, bearing either an H1- or a U6-hairpin siRNA casette, was used to inhibit expression of caspase-8 in HeLa-S3 cells (Tomar et al. 2003). Interestingly, in this study, only the U6-based cassette was effective at downregulating the levels of the target mRNA and corresponding protein. Retroviral vectors harbouring siRNA expression cassettes have also been described. In one study, an H1-siRNA cassette was placed either within the 3' long terminal repeat (LTR) of a self-inactivating retroviral vector (bearing a deletion in the $\mathrm{U} 3$ region of the $3^{\prime} \mathrm{LTR}$ ) or between the two LTRs (Barton \& Medzhitov 2002). The former construct had the advantage that the integrated provirus, derived from the viral vector, had an extra copy of the expression cassette in its 5' LTR. This was due to the process of reverse transcription, during which the $3^{\prime}$ LTR serves as a template for the generation of the $5^{\prime}$ LTR. Both types of constructs were successful at down-regulating endogenous p53 in mammalian cells lines. In another case, a retrovirus carrying an H1-based cassette successfully silenced a mutant (but not the wild-type) form of K-Ras in CAPAN-1 cells. The siRNA-treated cells lost their ability to cause tumours when injected into athymic nude mice (Brummelkamp et al. 2002b). Lentiviral vectors have also been used to transfer siRNA into cells. A self-inactivating lentiviral vector was created that expressed a hairpin siRNA targeted against enhanced green fluorescent protein (EGFP) under the control of the $\mathrm{Hl}$ promoter (Abbas-Terki et al. 2002). The vector was used to infect a cell line stably expressing EGFP and was found to successfully mediate silencing of EGFP expression. Remarkably, silencing persisted in the infected cells for at least 3 weeks from its onset. Lentiviral delivery of siRNA was also utilised to inhibit gene expression in vivo (Tiscornia et al. 2003). Mouse blastocysts, stably expressing EGFP, were transfected with a lentiviral vector expressing an anti-EGFP hairpin siRNA under the control of the $\mathrm{H} 1$ promoter. The successfully transfected blastocysts gave rise to mice in which EGFP expression was significantly reduced and, furthermore, the silencing effect was noted to persist in some of the progeny of these mice.

\section{Cell-type dependence on the efficiency and specificity of siRNA after transient or vector-based delivery}

In the large number of RNAi studies reported to date, siRNAs have been shown to be effective in a broad range of different cell types, from commonly used cell lines to primary cultured cells. However, 


\section{$\operatorname{siRNA}$}
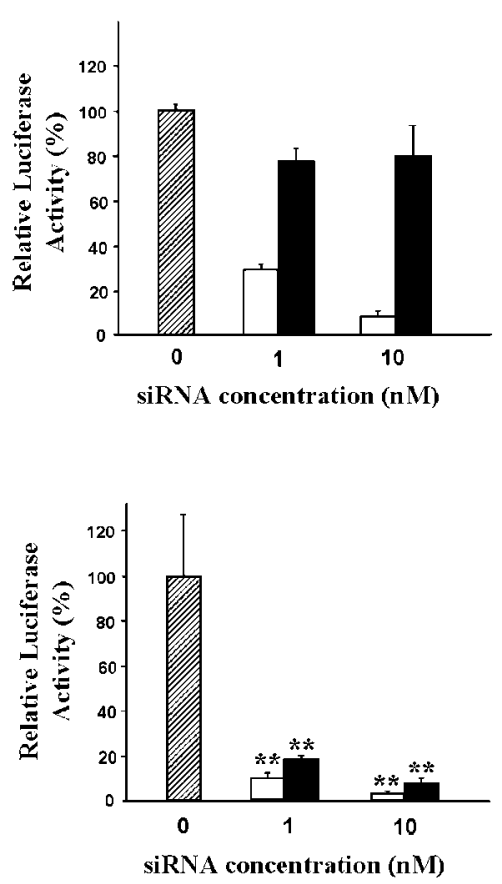

$\alpha$ T3

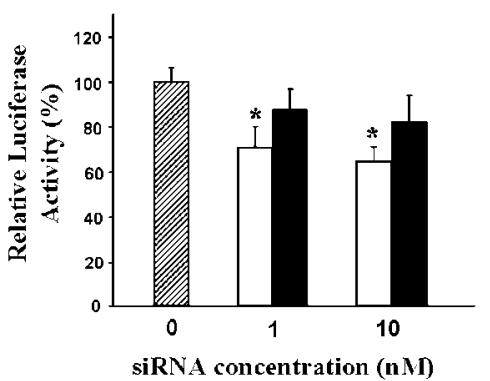

\section{Ad-U6-shRNA}
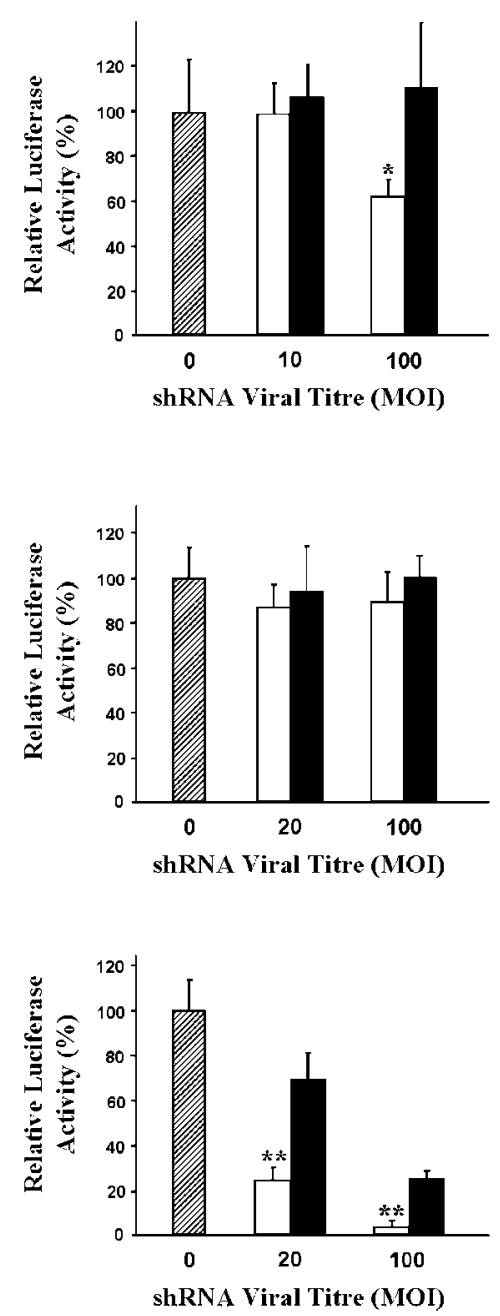

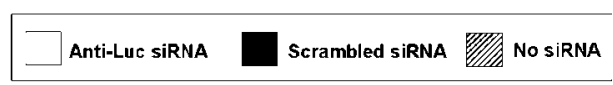

Figure 5 Comparison of the silencing efficacy of transiently and adenovirally delivered siRNA in different cell types. HeLa, PC-3 and $\alpha$ T3 cells were transfected with either in vitro transcribed anti-luciferase siRNA (using a lipid-based transfection reagent) or with an adenoviral vector expressing the corresponding anti-luciferase shRNA under the control of the U6 promoter (Ad-U6-shRNA). A scrambled (random sequence) siRNA (or shRNA) was used as a control. The cells were also transduced with an adenoviral vector expressing the target firefly luciferase (luc) mRNA. Forty-eight hours later, the cells were harvested and luciferase activity assayed. Data are presented as percentages (means \pm s.D.) of luciferase activity in the absence of siRNA or Ad-U6-shRNA $(n=4) .{ }^{*} P<0.05$ and ${ }^{* \star} P<0.001$ compared with scrambled siRNA ( $t$-test). 
typically siRNAs have been designed and tested in the single experimental cell line used for that study and the possibility that differing cellular environments may alter the efficacy of the siRNA-mediated silencing has not been extensively tested. To investigate this possibility six anti-luciferase siRNAs were designed and synthesised and tested in HeLa cells. The anti-luciferase siRNA most effective at inhibiting luciferase expression was then tested in a number of neuroendocrine cell lines. Furthermore, the same anti-luciferase siRNA sequence was used to make an shRNA, which was expressed via an adenoviral U6 vector. The results obtained (Fig. 5) show that the synthetically synthesised antiluciferase siRNA was highly effective at inhibiting luciferase activity when transfected into HeLa cells (transduced with the luciferase target). However, the presence of siRNA in PG-3 cells led to strong non-specific effects, as shown by the significant down-regulation of luciferase expression in the presence of the scrambled siRNA. In contrast, the transfection of the anti-luciferase siRNA into $\alpha \mathrm{T} 3$ cells caused extensive toxic effects and death within a 24-h period. This was true even at the lowest siRNA concentration used $(1 \mathrm{nM})$ and the effect was not due to the transfection reagent as toxic effects were almost absent in cells treated with the reagent only. Equally differing results were obtained when the anti-luciferase shRNA (note that this was the same sequence as the siRNA apart from the inclusion of the $9 \mathrm{bp}$ spacer sequence) under the control of the U6 promoter was delivered using an adenoviral vector. Figure 5 shows that, in this case, no inhibition of luciferase was seen in PC-3 cells, only modest inhibition of luciferase activity was obtained in HeLa cells and strong inhibition of luciferase was mediated in $\alpha \mathrm{T} 3$ by the active anti-luciferase shRNA and the shRNA scrambled control. Since adenoviral vectors can transduce all of these cell types with approximately equal efficiency, the difference in the results is unlikely to be due to shRNA expression levels. Instead, critical factors in determining the effectiveness of the shRNA may possibly be the concentration of Dicer enzyme (which may cleave the shRNA) and the IFN response (IFR) elicited by the adenoviral shRNA construct. If there was a significant IFR (where activation of the PKR pathway leads to indiscriminate down-regulation of gene expression) then PG-3 cells would appear especially prone to this non-specific effect, as similar concentrations of siRNA in other cell types have been shown in the past to mediate only specific silencing (e.g. Elbashir et al. 2001). The cellular level of Dicer and the IFR could also presumably influence the effectiveness of siRNA following transfection into the different cell types. However, in this case the differential efficiency of transfection and toxicity of the lipid-based reagents may also play major roles in determining the intracellular concentration of the siRNA and thereby its effectiveness.

Although these results have only been observed in a small number of cell lines they do highlight how the cellular environment can affect both the efficiency and specificity of shRNAs. Careful optimisation is therefore needed to achieve the desired balance between these two factors, while at the same time eliminate potential toxic effects caused by increased concentration of the siRNA in the cell.

\section{Conclusion}

RNA interference and related phenomena began to be studied as a part of the biology of mainly lower organisms (such as Neurospora or C. elegans) or plants. Subsequently, however, it was discovered that equivalent cellular pathways exist in mammalian cells. As the study of these pathways progressed, it was soon evident that RNAi, more specifically, siRNA could be utilised as a tool to study mammalian gene function as it allowed downregulation of gene expression, hence providing a simple alternative to creating cell lines or transgenic animals with knock-out phenotypes. To facilitate these studies of gene function, a number of techniques aimed at easing the synthesis and screening of synthetic siRNA have been developed. Examples include the T7-driven in vitro transcription of siRNAs and the development of prescreened siRNA libraries. With the advent of vector-based systems for the expression of shRNA, many of the problems associated with siRNA delivery, e.g. low transfection efficiency and inappropriate subcellular localisation, have also begun to be solved. In parallel, viral vector-based systems allow the silencing effect of the shRNA to be prolonged and used in animal in vivo models. However, for RNAi technology to reach its full potential as a scientific tool and possibly be used for 
therapeutic purposes, a mechanism to avoid the non-specific silencing effects and activation of the IFN response must be found.

\section{Acknowledgements}

We would like to thank the Human Frontiers Science Programme for funding this work.

\section{References}

Abbas-Terki T, Blanco-Bose W, Deglon N, Pralong W \& Aebischer P 2002 Lentiviral-mediated RNA interference. Human Gene Therapy 13 2197-2201.

Barton GM \& Medzhitov R 2002 Retroviral delivery of small interfering RNA into primary cells. PNAS 99 14943-14945.

Berns K, Hijmans EM, Mullenders J, Brummelkamp TR, Velds A, Heimerikx M, Kerkhoven RM, Madiredjo M, Nijkamp W, Weigelt B, Agami R, Ge W, Cavet G, Linsley PS, Beijersbergen RL \& Bernards R 2004 A large-scale RNAi screen in human cells identifies new components of the p53 pathway. Nature $\mathbf{4 2 8}$ 431-437.

Bernstein E, Caudy AA, Hammond SM \& Hannon GJ 2001 Role for a bidentate ribonuclease in the initiation step of RNA interference. Nature 409 363-366.

Boden D, Pusch O, Silbermann R, Lee F, Tucker L \& Ramratnam B 2004 Enhanced gene silencing of HIV-1 specific siRNA using microRNA designed hairpins. Nucleic Acids Research 32 1154-1158.

Bridge AJ, Pebernard S, Ducraux A, Nicoulaz AL \& Iggo R 2003 Induction of an interferon response by RNAi vectors in mammalian cells. Nature Genetics 34 263-264.

Brummelkamp TR, Bernards R \& Agami R 2002a A system for stable expression of short interfering RNAs in mammalian cells. Science 296 550-553.

Brummelkamp TR, Bernards R \& Agami R $2002 b$ Stable suppression of tumorigenicity by virus-mediated RNA interference. Cancer Cell 2 243-247.

Capodici J, Kariko K \& Weissman D 2002 Inhibition of HIV-1 infection by small interfering RNA-mediated RNA interference. fournal of Immunology 169 5196-5201.

Chiu YL \& Rana TM 2002 RNAi in human cells: basic structural and functional features of small interfering RNA. Molecular Cell $\mathbf{1 0}$ 549-561

Cogoni C \& Macino G 1999 Gene silencing in Neurospora crassa requires a protein homologous to RNA-dependent RNA polymerase. Nature 399 166-169.

Conrad C \& Rauhut R 2002 Ribonuclease III: new sense from nuisance. International Fournal of Biochemistry and Cell Biology $\mathbf{3 4}$ $116-129$.

Dalmay T, Hamilton A, Rudd S, Angell S \& Baulcombe DC 2000 An RNA-dependent RNA polymerase gene in Arabidopsis is required for posttranscriptional gene silencing mediated by a transgene but not by a virus. Cell $101543-553$.

Doench JG, Petersen CP \& Sharp PA 2003 siRNAs can function as miRNAs. Genes and Development 17 438-442.

Donze O \& Picard D 2002 RNA interference in mammalian cells using siRNAs synthesized with T7 RNA polymerase. Nucleic Acids Research 30 e 46

Elbashir SM, Lendeckel W \& Tuschl T 2001 RNA interference is mediated by 21- and 22-nucleotide RNAs. Genes and Development 15 188-200.
Elbashir SM, Harborth J, Weber K \& Tuschl T 2002 Analysis of gene function in somatic mammalian cells using small interfering RNAs. Methods 26 199-213.

Golden TA, Schauer SE, Lang JD, Pien S, Mushegian AR, Grossniklaus U, Meinke DW \& Ray A 2002 SHORT INTEGUMENTS1/SUSPENSOR1/CARPEL FACTORY, a Dicer homolog, is a maternal effect gene required for embryo development in Arabidopsis. Plant Physiology 130 808-822.

Grishok A, Pasquinelli AE, Conte D, Li N, Parrish S, Ha I, Baillie DL, Fire A, Ruvkun G \& Mello CC 2001 Genes and mechanisms related to RNA interference regulate expression of the small temporal RNAs that control C. elegans developmental timing. Cell $10623-34$

Grosshans H \& Slack FJ 2002 Micro-RNAs: small is plentiful. fournal of Cell Biology 156 17-21.

Hammond SM, Bernstein E, Beach D \& Hannon GJ 2000 An RNA-directed nuclease mediates post-transcriptional gene silencing in Drosophila cells. Nature 404 293-296.

Hsieh AC, Bo R, Manola J, Vazquez F, Bare O, Khvorova A, Scaringe S \& Sellers WR 2004 A library of siRNA duplexes targeting the phosphoinositide 3-kinase pathway: determinants of gene silencing for use in cell-based screens. Nucleic Acids Research 32 893-901.

Hutvagner G \& Zamore PD 2002 A microRNA in a multipleturnover RNAi enzyme complex. Science 297 2056-2060.

Hutvagner G, McLachlan J, Pasquinelli AE, Balint E, Tuschl T \& Zamore PD 2001 A cellular function for the RNA-interference enzyme Dicer in the maturation of the let-7 small temporal RNA. Science 293 834-838.

Jackson AL, Bartz SR, Schelter J, Kobayashi SV, Burchard J, Mao M, Li B, Cavet G \& Linsley PS 2003 Expression profiling reveals off-target gene regulation by RNAi. Nature Biotechnology 21 635-637.

Ketting RF, Haverkamp TH, van Luenen HG \& Plasterk RH 1999 Mut-7 of C. elegans, required for transposon silencing and RNA interference, is a homolog of Werner syndrome helicase and RNaseD. Cell 99 133-141.

Ketting RF, Fischer SE, Bernstein E, Sijen T, Hannon GJ \& Plasterk RH 2001 Dicer functions in RNA interference and in synthesis of small RNA involved in developmental timing in $C$ elegans. Genes and Development 15 2654-2659.

Khvorova A, Reynolds A \& Jayasena SD 2003 Functional siRNAs and miRNAs exhibit strand bias. Cell 115 209-216.

Kim DH, Longo M, Han Y, Lundberg P, Cantin E \& Rossi JJ 2004 Interferon induction by siRNAs and ssRNAs synthesized by phage polymerase. Nature Biotechnology 22 321-325.

Lagos-Quintana M, Rauhut R, Lendeckel W \& Tuschl T 2001 Identification of novel genes coding for small expressed RNAs. Science 294 853-858.

Lau NC, Lim LP, Weinstein EG \& Bartel DP 2001 An abundant class of tiny RNAs with probable regulatory roles in Caenorhabditis elegans. Science 294 858-862.

Lee NS, Dohjima T, Bauer G, Li H, Li MJ, Ehsani A, Salvaterra P \& Rossi J 2002 Expression of small interfering RNAs targeted against HIV-1 rev transcripts in human cells. Nature Biotechnology 20 500-505.

Lee RC, Feinbaum RL \& Ambros V 1993 The C. elegans heterochronic gene lin-4 encodes small RNAs with antisense complementarity to lin-14. Cell $\mathbf{7 5} 843-854$.

Luo B, Heard AD \& Lodish HF 2004 Small interfering RNA production by enzymatic engineering of DNA (SPEED). PNAS $1015494-5499$.

Manche L, Green SR, Schmedt C \& Mathews MB 1992 Interactions between double-stranded RNA regulators and the protein kinase DAI. Molecular and Cellular Biology 12 5238-5248. 
Martens H, Novotny J, Oberstrass J, Steck TL, Postlethwait P \& Nellen W 2002 RNAi in Dictyostelium: the role of RNA-directed RNA polymerases and double-stranded RNase. Molecular Biology of the Cell 13 445-453.

Martinez J, Patkaniowska A, Urlaub H, Luhrmann R \& Tuschl T 2002 Single-stranded antisense siRNAs guide target RNA cleavage in RNAi. Cell 110 563-574.

Miller VM, Xia H, Marrs GL, Gouvion CM, Lee G, Davidson BL \& Paulson HL 2003 Allele-specific silencing of dominant disease genes. PNAS $1007195-7200$.

Miyagishi M \& Taira K 2003 Strategies for generation of an siRNA expression library directed against the human genome. Oligonucleotides 13 325-333.

Miyagishi M, Matsumoto S \& Taira K 2004 Generation of an shRNAi expression library against the whole human transcripts. Virus Research 102 117-124.

Mourelatos Z, Dostie J, Paushkin S, Sharma A, Charroux B, Abel L, Rappsilber J, Mann M \& Dreyfuss G 2002 miRNPs: a novel class of ribonucleoproteins containing numerous microRNAs. Genes and Development 16 720-728.

Mourrain P, Beclin C, Elmayan T, Feuerbach F, Godon C, Morel JB, Jouette D, Lacombe AM, Nikic S, Picault N, Remoue K, Sanial M, Vo TA \& Vaucheret H 2000 Arabidopsis SGS2 and SGS3 genes are required for posttranscriptional gene silencing and natural virus resistance. Cell 101 533-542.

Nicholson RH \& Nicholson AW 2002 Molecular characterization of a mouse cDNA encoding Dicer, a ribonuclease III ortholog involved in RNA interference. Mammalian Genome 13 67-73.

Nykanen A, Haley B \& Zamore PD 2001 ATP requirements and small interfering RNA structure in the RNA interference pathway. Cell 107 309-321.

Olsen PH \& Ambros V 1999 The lin-4 regulatory RNA controls developmental timing in Caenorhabditis elegans by blocking LIN-14 protein synthesis after the initiation of translation. Developmental Biology 216 671-680.

Provost P, Dishart D, Doucet J, Frendewey D, Samuelsson B \& Radmark O 2002a Ribonuclease activity and RNA binding of recombinant human Dicer. EMBO Journal $215864-5874$.

Provost P, Silverstein RA, Dishart D, Walfridsson J, Djupedal I, Kniola B, Wright A, Samuelsson B, Radmark O \& Ekwall K $2002 b$ Dicer is required for chromosome segregation and gene silencing in fission yeast cells. PNAS 99 16648-16653.

Reinhart BJ, Slack FJ, Basson M, Pasquinelli AE, Bettinger JC, Rougvie AE, Horvitz HR \& Ruvkun G 2000 The 21-nucleotide let-7 RNA regulates developmental timing in Caenorhabditis elegans. Nature 403 901-906.

Reynolds A, Leake D, Boese Q, Scaringe S, Marshall WS \& Khvorova A 2004 Rational siRNA design for RNA interference. Nature Biotechnology 22 326-330.

Schramke V \& Allshire R 2003 Hairpin RNAs and retrotransposon LTRs effect RNAi and chromatin-based gene silencing. Science 301 1069-1074.

Schwarz DS, Hutvagner G, Du T, Xu Z, Aronin N \& Zamore PD 2003 Asymmetry in the assembly of the RNAi enzyme complex. Cell 115 199-208.

Sen G, Wehrman TS, Myers JW \& Blau HM 2004 Restriction enzyme-generated siRNA (REGS) vectors and libraries. Nature Genetics 36 183-189.

Shen C, Buck AK, Liu X, Winkler M \& Reske SN 2003 Gene silencing by adenovirus-delivered siRNA. FEBS Letters 539 $111-114$.
Shirane D, Sugao K, Namiki S, Tanabe M, Iino M \& Hirose K 2004 Enzymatic production of RNAi libraries from cDNAs. Nature Genetics 36 190-196.

Sijen T, Fleenor J, Simmer F, Thijssen KL, Parrish S, Timmons L, Plasterk RH \& Fire A 2001 On the role of RNA amplification in dsRNA-triggered gene silencing. Cell 107 465-476.

Sledz CA, Holko M, de Veer MJ, Silverman RH \& Williams BR 2003 Activation of the interferon system by short-interfering RNAs. Nature Cell Biology 5834 -839.

Smardon A, Spoerke JM, Stacey SC, Klein ME, Mackin N \& Maine EM 2000 EGO-1 is related to RNA-directed RNA polymerase and functions in germ-line development and RNA interference in C. elegans. Current Biology 10 169-178.

Sohail M, Doran G, Riedemann J, Macaulay V \& Southern EM 2003 A simple and cost-effective method for producing small interfering RNAs with high efficacy. Nucleic Acids Research 31 e38.

Tabara H, Yigit E, Siomi H \& Mello CC 2002 The dsRNA binding protein RDE-4 interacts with RDE-1, DCR-1, and a DExH-box helicase to direct RNAi in C. elegans. Cell 109 861-871.

Tiscornia G, Singer O, Ikawa M \& Verma IM 2003 A general method for gene knockdown in mice by using lentiviral vectors expressing small interfering RNA. PNAS 100 1844-1848.

Tomar RS, Matta H \& Chaudhary PM 2003 Use of adeno-associated viral vector for delivery of small interfering RNA. Oncogene 22 5712-5715.

Ui-Tei K, Naito Y, Takahashi F, Haraguchi T, Ohki-Hamazaki H, Juni A, Ueda R \& Saigo K 2004 Guidelines for the selection of highly effective siRNA sequences for mammalian and chick RNA interference. Nucleic Acids Research 32 936-948.

Volpe TA, Kidner C, Hall IM, Teng G, Grewal SI \& Martienssen RA 2002 Regulation of heterochromatic silencing and histone H3 lysine-9 methylation by RNAi. Science 297 1833-1837.

van de Wetering M, Oving I, Muncan V, Pon Fong MT, Brantjes $\mathrm{H}$, van Leenen D, Holstege FG, Brummelkamp TR, Agami R \& Clevers H 2003 Specific inhibition of gene expression using a stably integrated, inducible small-interfering-RNA vector. $E M B O$ Reports 4 609-615.

Xia H, Mao Q Paulson HL \& Davidson BL 2002 siRNA-mediated gene silencing in vitro and in vivo. Nature Biotechnology 20 1006-1010.

Xia XG, Zhou H, Ding H, Affar eB, Shi Y \& Xu Z 2003 An enhanced U6 promoter for synthesis of short hairpin RNA. Nucleic Acids Research $\mathbf{3 1}$ e100.

Zamore PD, Tuschl T, Sharp PA \& Bartel DP 2000 RNAi: double-stranded RNA directs the ATP-dependent cleavage of mRNA at 21 to 23 nucleotide intervals. Cell $10125-33$.

Zeng Y, Yi R \& Cullen BR 2003 MicroRNAs and small interfering RNAs can inhibit mRNA expression by similar mechanisms. PNAS 100 9779-9784.

Zhang H, Kolb FA, Brondani V, Billy E \& Filipowicz W 2002 Human Dicer preferentially cleaves dsRNAs at their termini without a requirement for ATP. EMBO Joumal 21 5875-5885.

Received 5 May 2004

Accepted 14 July 2004

Made available online as an Accepted Preprint 26 July 2004 